\title{
Caracterización de problemas gástricos en usuarios de un hospital de Huancavelica, Perú
}

\author{
Characterization of gastric problems in users of a hospital in Huancavelica, Peru
}

Lilia Maria Nieva-Villegasª*, Elsy Sara Carhuachuco-Rojas²,b , Walter Edgar Gómez-Gonzales ${ }^{3, c}$

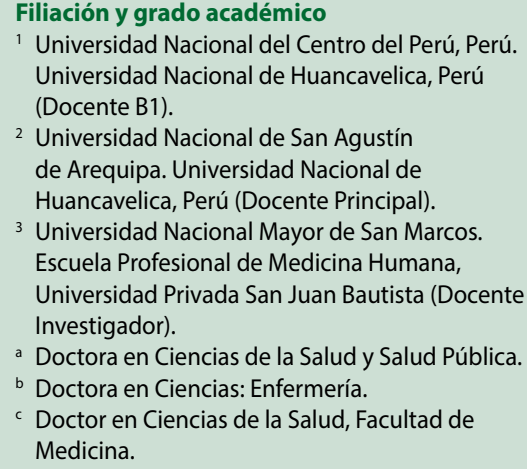

Doctora en Ciencias de la Salud y Salud Pública.

b Doctora en Ciencias: Enfermería.

Doctor en Ciencias de la Salud, Facultad de Medicina.

(1) ORCID iD de Lilia Nieva-Villegas https://orcid.org/0000-0001-9889-6332 D. ORCID iD de Elsy Carhuachuco-Rojas https://orcid.org/0000-0002-6562-1305

10. ORCID iD de Walter Gómez-Gonzales https://orcid.org/0000-0003-0706-7614

Contribución de los autores

LNV: contribución de idea de investigación, recolección de datos, análisis de datos, redacción del artículo, revisión de la versión final.

ECR: contribución de idea de investigación, recolección de datos, análisis de datos, redacción del artículo, revisión de la versión final. WGG: contribución de revisión del artículo, aprobación de la versión final del artículo. Fuentes de financiamiento FAEDI (Fondo de apoyo Económico a Docentes Investigadores) de la Universidad Nacional de Huancavelica.

Conflictos de interés

WGG: forma parte del equipo editorial de la Revista Peruana de Ciencias de la Salud, reservándose de participar en el proceso de revisión.

Recibido: 29-01-2020

Arbitrado por pares

Aceptado: 07-05-2020

Citar como

Nieva-Villegas LM, Carhuachuco-Rojas ES, GómezGonzales WE. Caracterización de problemas gástricos en usuarios de un hospital de Huancavelica, Perú. Rev Peru Cienc Salud. 2020; 2(2): 82-6. doi: https://doi.org/10.37711/rpcs.2020.2.2.124

Correspondencia

Lilia María Nieva-Villegas

Dirección: Jr. Santa Cecilia N 195. Huancayo,

Junín, Perú

Cel.: 917180217

Email: lilianievav@gmail.com

\section{RESUMEN}

Objetivo. Caracterizar los problemas gástricos de la población usuaria del Hospital Regional de Huancavelica Zacarias Correa Valdivia en los años 2015-2017. Métodos. Fue un estudio de diseño descriptivo, transversal, no experimental, retrospectivo y bibliográfico, realizándose la revisión bibliográfica del Sistema de Información en Salud (HIS) y de historias clínicas de pacientes atendidos con problemas gástricos en los años mencionados ( $n=3261$ ). Resultados. Se evidenció que el $67 \%$ de la población son mujeres; 34,7 \% nació en la región de Huancavelica, 93,7 \% tuvo trabajo de oficina, el 78,1\% era usuarios del servicio de gastroenterología, 93,7 \%, no informó su estado de alta, el $34,5 \%$, no poseía un sistema de seguro de salud y el $61,7 \%$ de la población padeció gastritis crónica inespecífica. Los pacientes diagnosticados con problemas gástricos representaban en el 2015 un 64,9\% de casos, en el 2016 un 55,4 \% y en el 2017 un 64,3 \% del total de consultas. Conclusión. El género femenino presentó la mayor frecuencia de diagnóstico de gastritis crónica no específica, así como los jóvenes y adultos jóvenes para el año 2017 según el total de diagnósticos observados retrospectivamente en el reporte HIS.

Palabras clave: estudios transversales, estudios retrospectivos, gastroenterología, alta del paciente, recurrente epitelial, distrofias corneales, hereditarias, seguro, registros médicos, remisión y consulta, gastritis (Fuente: DeCS - BIREME).

\section{ABSTRACT}

Objective. To characterize the gastric problems of user population of the Regional Hospital of Huancavelica Zacarias Correa Valdivia in the years 2015-2017. Method. It is a descriptive, cross-sectional, non-experimental, retrospective and bibliographic study, with a bibliographic review of the Health Information System (HIS) and medical records of patients treated with gastric problems in the years mentioned $(n=3261)$. Results. It was evidenced that $67 \%$ of the population are women. $34.7 \%$ were born in the Huancavelica region. $93.7 \%$ had office work. $78.1 \%$ were users of the gastroenterology service. $93.7 \%$ did not report their discharge status. $34.5 \%$ did not have a health insurance system, and $61.7 \%$ of the population suffered from nonspecific chronic gastritis. Patients diagnosed with gastric problems represented $64.9 \%$ of cases in 2015, 55.4\% in 2016 and $64.3 \%$ of total consultations in 2017. Conclusion. Women presented the highest frequency of diagnosis of chronic non-specific gastritis, as well as young people and young adults for the year 2017 according to the total number of diagnoses retrospectively observed in the HIS report

Keywords: cross-sectional studies, retrospective studies, gastroenterology, patient discharge, epithelial recurrent, corneal dystrophies, hereditary, insurance, medical records, referral and consultation, gastritis (Source: MeSH-NLM). 


\section{INTRODUCCIÓN}

Las enfermedades gástricas son uno de los principales problemas de salud pública mundial ${ }^{(1)}$, que se transmiten por vía fecal - oral, o bien por consumo de agua y alimentos contaminados. En conjunto, se trata de un problema que afecta a todas personas de cualquier edad o condición social, En conjunto, se trata de un problema que afecta en el nivel socioeconómico bajo ${ }^{(2,3)}$.

La elevada incidencia de estas, además de su asociación con padecimientos de alta morbilidad, como las gastritis crónicas, las convierten en un problema de salud pública en nuestro país que requiere mucha atención ${ }^{(1)}$. Asimismo, su alta prevalencia intrafamiliar en otros países, nos lleva a pensar que es el núcleo familiar es, precisamente, fuente común de infección y reinfección ${ }^{(4)}$. En el Perú, estas enfermedades gástricas están relacionadas a la presencia de la infección por $H$. pylori, por ser considerada la causa principal de la gastritis crónica, además de estar considerado como un factor carcinogénico. Asimismo, se relacionan al cáncer gástrico, que es considerado la principal causa de muerte para ambos sexos.

Estos hechos nos llevan a puntualizar que existe gran variabilidad de patógenos que afectan a diferentes grupos de población en diferentes zonas; lo que indica que existen probablemente también diferentes cepas ${ }^{(3)}$. Por lo que el conocimiento sobre la epidemiología en diversas poblaciones, especialmente en zonas de alta incidencia, se hace necesario. Su detección temprana nos ayudará para poder suministrar un tratamiento adecuado a tiempo, evitando y disminuyendo el problema a nivel de riesgo ${ }^{(5)}$; para poder suministrar un tratamiento adecuado a tiempo, evitando la morbimortalidad y sus complicaciones ${ }^{(6)}$.

Por tal motivo, es importante conocer el estado actual de las enfermedades gástricas en la población usuaria del Hospital Regional de Huancavelica Zacarías Correa Valdivia, por ser, un hospital de referencia en la provincia de Huancavelica. Se hizo con el objeto de conocer la situación real en la zona, la asociación intrafamiliar como factor de riesgo para la trasmisión; ya que en la provincia de Huancavelica no se han encontrado estudios al respecto. En todo caso, la idea fue poder estar en condiciones de compararlos resultados con otras zonas del país y, a partir de ahí, dar paso a otros niveles de investigación relacionadas al objeto de estudio.

\section{MÉTODOS}

Se desarrolló mediante un diseño no experimental, longitudinal, descriptivo y retrospectivo. La población de la investigación estuvo compuesta por los usuarios del Hospital, Regional de Huancavelica durante los años 2015-2017 y la muestra, estuvo constituida por el reporte HIS y las historias clínicas de los pacientes diagnosticados con problemas gástricos que acudieron a una consulta médica entre los años 2015 y 2017. Se recolectó la tabla de datos del sistema de reportes de HIS, con el filtro de Código Internacional de Enfermedades (CIE), relacionados a problemas gástricos y se realizó la revisión de historias clínicas para la recolección de

Tabla 1. Características socio-institucionales de la población que presenta enfermedades gástricas en el Hospital Regional Zacarías Correa Valdivia Huancavelica

\begin{tabular}{|c|c|c|c|}
\hline $\begin{array}{l}\text { Características } \\
\text { Socio-institucionales }\end{array}$ & Categorías & fi & $\%$ \\
\hline \multirow{2}{*}{ Género del paciente } & Masculino & 1075 & 33,0 \\
\hline & Femenino & 2186 & 67,0 \\
\hline \multirow{9}{*}{$\begin{array}{l}\text { Provincia de } \\
\text { procedencia }\end{array}$} & Acobamba & 5 & 0,2 \\
\hline & Castrovirreyna & 1 & 0,0 \\
\hline & Huancavelica & 1132 & 34,7 \\
\hline & Huaytará & 2 & 0,1 \\
\hline & Tayacaja & 1 & 0,0 \\
\hline & Angaraes & 6 & 0,2 \\
\hline & Lima & 1 & 0,0 \\
\hline & Pisco & 1 & 0,0 \\
\hline & No informa & 2112 & 64,8 \\
\hline \multirow{3}{*}{ Origen de la atención } & Hospital & 28 & 0,9 \\
\hline & Emergencia & 176 & 5,4 \\
\hline & Consultorio & 3057 & 93,7 \\
\hline \multirow{13}{*}{$\begin{array}{l}\text { Servicio de } \\
\text { procedencia }\end{array}$} & Medicina general & 245 & 7,5 \\
\hline & Cirugía & 70 & 2,1 \\
\hline & Emergencia & 176 & 5,4 \\
\hline & Cardiología & 13 & 0,4 \\
\hline & Gastroenterología & 2548 & 78,1 \\
\hline & Psicología & 1 & 0,0 \\
\hline & Urología & 1 & 0,0 \\
\hline & Medicina interna & 156 & 4,8 \\
\hline & Endocrinología & 2 & 0,1 \\
\hline & Ginecología & 3 & 0,1 \\
\hline & Infectología & 1 & 0,0 \\
\hline & Oftalmología & 41 & 1,3 \\
\hline & Medicina de rehabilitación & 4 & 0,1 \\
\hline \multirow{4}{*}{ Condición de alta } & Alta médica & 193 & 5,9 \\
\hline & Hospitalización & 9 & 0,3 \\
\hline & Fuga & 2 & 0,1 \\
\hline & No informa & 3057 & 93,7 \\
\hline \multirow{4}{*}{ Sistema de seguro } & Seguro Integral de Salud & 450 & 13,8 \\
\hline & Usuario & 1126 & 34,5 \\
\hline & Militar & 5 & 0,2 \\
\hline & No informa & 1680 & 51,5 \\
\hline
\end{tabular}


datos sociodemográficos de los usuarios identificados. Para el procesamiento y análisis de los datos se utilizó el paquete estadístico IBM Statistics para Windows Vers. 24,0, además de la hoja de cálculo Microsoft Office Excel 2016. También se utilizó la estadística descriptiva: tablas y gráficos estadísticos.

El Hospital Regional de Huancavelica, tiene un nivel II de complejidad, se encuentra ubicado en el distrito de Huancavelica, cuenta con una población designada de 24455 habitantes y atiende a una población sociodemográfica heterogénea ya que es un hospital de referencia. El Hospital, mantiene una importante incidencia de infecciones respiratorias y diarreicas, debido a lo cual se sitúa en un escenario epidemiológico particular que requiere una eficiente respuesta de nuestros servicios; especialmente si tenemos en cuenta que nuestra demanda en los diferentes servicios es creciente.

\section{RESULTADOS}

En los 3261 casos, se evidenció un predominio de problemas gástricos en el género femenino (67\%) provenientes de la misma región de Huancavelica (34,7 \%). La mayoría de los casos de problemas gástricos procedían de los servicios de consultorio (93,7\%); específicamente del servicio de gastroenterología $(78,1 \%)$. En la historia clínica no se informaba la condición del alta $(93,7 \%)$ y los usuarios no contaban con sistema de seguro $(34,5 \%)$ (ver tabla 1).
Se evidencia un incremento significativo en la incidencia de casos de problemas gástricos secuencialmente en cada año; además, este incremento anual se mantiene en casos específicos, como gastritis. Asimismo, se observa que la población usuaria presenta con mayor frecuencia gastritis crónica no específica que, según criterios diagnósticos clínicos evidenciados en las historias clínicas, fue del $64,9 \%$ en el año 2015 ; del $55,4 \%$ en el 2016 ; y del 64,3\% en el 2017 (ver tabla 2).

La tasa de gastritis crónica no especifica tiene una mayor incidencia en el género femenino (62,9\%); mientras que los principales casos de problemas gástricos en el género masculino fueron ocasionados por la gastritis crónica específica (ver tabla 3).

\section{DISCUSIÓN}

Con respecto a la prevalencia mundial de problemas gástricos, los estudios estiman que, en la actualidad, aproximadamente el $50 \%$ de la población mundial tiene algún problema gástrico, aunque la mayoría nunca presente síntomas clínicos ${ }^{(7)}$. Es importante destacar, además, con respecto a la prevalencia de los problemas gástricos según el sexo de los usuarios, una publicación boliviana del 2013 que encontró una prevalencia de infección por $\mathrm{H}$. pylori del $62,9 \%$, predominante en el sexo masculino (65\%) en relación al sexo femenino $(62,7 \%)^{(8)}$. Sin embargo, otros resultados evidencian una prevalencia de problemas gástricos mayor en mujeres

Tabla 2. Incidencia de problemas gástricos por años, en el Hospital Regional Zacarías Correa Valdivia, Huancavelica

\begin{tabular}{|c|c|c|c|c|c|c|c|c|}
\hline \multirow{3}{*}{$\begin{array}{c}\text { CODIGO } \\
\text { CIE-10 }\end{array}$} & \multicolumn{6}{|c|}{ Años de presentación } & \multirow{2}{*}{\multicolumn{2}{|c|}{ Total }} \\
\hline & \multicolumn{2}{|c|}{2015} & \multicolumn{2}{|c|}{2016} & \multicolumn{2}{|c|}{2017} & & \\
\hline & $f i$ & $\%$ & $f i$ & $\%$ & $f i$ & $\%$ & $f i$ & $\%$ \\
\hline K295 & 523 & 64,9 & 560 & 55,4 & 929 & 64,3 & 2012 & 61,7 \\
\hline K297 & 112 & 13,9 & 282 & 27,9 & 361 & 25,0 & 755 & 23,2 \\
\hline K291 & 43 & 5,3 & 31 & 3,1 & 84 & 5,8 & 158 & 4,8 \\
\hline K293 & 45 & 5,6 & 85 & 8,4 & 8 & 0,6 & 138 & 4,2 \\
\hline K290 & 51 & 6,3 & 45 & 4,5 & 24 & 1,7 & 120 & 3,7 \\
\hline K296 & 3 & 0,4 & 3 & 0,3 & 23 & 1,6 & 29 & 0,9 \\
\hline K259 & 13 & 1,6 & 0 & 0,0 & 0 & 0,0 & 13 & 0,4 \\
\hline K294 & 3 & 0,4 & 2 & 0,2 & 7 & 0,5 & 12 & 0,4 \\
\hline K250 & 7 & 0,9 & 0 & 0,0 & 0 & 0,0 & 7 & 0,2 \\
\hline K292 & 2 & 0,2 & 3 & 0,3 & 2 & 0,1 & 7 & 0,2 \\
\hline K299 & 0 & 0,0 & 0 & 0,0 & 5 & 0,3 & 5 & 0,2 \\
\hline $\mathrm{C} 161$ & 1 & 0,1 & 0 & 0,0 & 0 & 0,0 & 1 & 0,0 \\
\hline K298 & 0 & 0,0 & 0 & 0,0 & 1 & 0,1 & 1 & 0,0 \\
\hline K253 & 1 & 0,1 & 0 & 0,0 & 0 & 0,0 & 1 & 0,0 \\
\hline K254 & 1 & 0,1 & 0 & 0,0 & 0 & 0,0 & 1 & 0,0 \\
\hline K257 & 1 & 0,1 & 0 & 0,0 & 0 & 0,0 & 1 & 0,0 \\
\hline
\end{tabular}


Tabla 3. Frecuencia de problemas gástricos según genero del usuario, en el Hospital Regional Zacarías Correa Valdivia, Huancavelica

\begin{tabular}{|c|c|c|c|c|c|c|}
\hline \multirow{3}{*}{ CIE- 10} & \multicolumn{4}{|c|}{ Sexo } & \multirow{2}{*}{\multicolumn{2}{|c|}{ Total }} \\
\hline & \multicolumn{2}{|c|}{ Masculino } & \multicolumn{2}{|c|}{ Femenino } & & \\
\hline & fi & $\%$ & fi & $\%$ & fi & $\%$ \\
\hline K295 & 637 & 59,3 & 1375 & 62,9 & 2012 & 61,7 \\
\hline K297 & 242 & 22,5 & 513 & 23,5 & 755 & 23,2 \\
\hline K291 & 58 & 5,4 & 100 & 4,6 & 158 & 4,8 \\
\hline K293 & 54 & 5,0 & 84 & 3,8 & 138 & 4,2 \\
\hline K290 & 45 & 4,2 & 75 & 3,4 & 120 & 3,7 \\
\hline K296 & 11 & 1,0 & 18 & 0,8 & 29 & 0,9 \\
\hline K259 & 10 & 0,9 & 3 & 0,1 & 13 & 0,4 \\
\hline K294 & 4 & 0,4 & 8 & 0,4 & 12 & 0,4 \\
\hline K250 & 5 & 0,5 & 2 & 0,1 & 7 & 0,2 \\
\hline K292 & 4 & 0,4 & 3 & 0,1 & 7 & 0,2 \\
\hline K299 & 2 & 0,2 & 3 & 0,1 & 5 & 0,2 \\
\hline C161 & 0 & 0,0 & 1 & 0,0 & 1 & 0,0 \\
\hline K253 & 1 & 0,1 & 0 & 0,0 & 1 & 0,0 \\
\hline K254 & 1 & 0,1 & 0 & 0,0 & 1 & 0,0 \\
\hline K257 & 1 & 0,1 & 0 & 0,0 & 1 & 0,0 \\
\hline K298 & 0 & 0,0 & 1 & 0,0 & 1 & 0,0 \\
\hline
\end{tabular}

$(47,1 \%)$ que en varones $(42,1 \%)$ aunque sin diferencia estadísticamente significativamente ${ }^{(4)}$. Mientras, otra investigación halló una prevalencia de $71,2 \%$ en varones y $69,1 \%$ en mujeres $(p=0,23)$ con gastritis crónica activa de $1985-2002^{(9-11)}$. Otro estudio muestra evidencias para un tipo distinto de diferencias, dado que encuentra una mayor prevalencia en los varones de falta de higiene durante la infancia y la juventud ${ }^{(12)}$. Por consiguiente, en la mayoría de estos estudios no existe una diferencia estadísticamente significativa con respecto a la prevalencia de problemas gástricos entre ambos sexos, tal y como se evidencia en los resultados de esta investigación.

Con respecto al grupo etario, la investigación boliviana del 2013 manifiesta que existe una prevalencia mayor en el grupo etario de 14 a 29 años $(57,2 \%)^{(7)}$. En la investigación de Lima, del 2016, se evidencia el inicio de cierta prevalencia en menores de 15 años $(36,3 \%)$. Se cree que los cambios de estilo de vida y mejoras sanitarias de zonas de clase media y rica estén modificando la prevalencia y edad de adquisición de la infección en la infancia ${ }^{(5,13)}$, sin embargo, la prevalencia de estos casos es alta en zonas pobres, como los de la región de Huancavelica, los cuales se incrementan por posibles reinfecciones en la etapa adulta ${ }^{(14)}$. Cabe agregar, que una de las limitaciones de la investigación fue la fragmentación del Sistema Nacional de salud, concretamente, la fragmentación del sistema de información y la dificultad de caracterizar problemas de salud de forma más integral, permitiendo mostrar un mejor panorama de la situación de salud que permita focalizar adecuadamente las políticas de salud a fin de que estas incidan positivamente en la salud de la población.

Se concluye indicando que a pesar de la mayor incidencia y cronicidad de los problemas gástricos, como la gastritis crónica no especifica en ambos géneros y los diferentes grupos etarios, no se hace incidencia en la necesidad de diagnóstico precoz y su relación con la bacteria $H$. pilory, una de las más comunes en este tipo de enfermedades. Por tal motivo, se recomienda continuar las investigaciones enfocadas en la relación de esta alta incidencia y la presencia de H. pylori; Asimismo, se recomienda al Hospital Regional de Huancavelica una adecuada sistematización de la información concerniente a las atenciones para garantizar el desarrollo de estudios de interés y la toma de decisiones. Por último, se exhorta a todos los profesionales de la salud para que otorguen una mayor prioridad en la prevención primaria y secundaria sobre las patologías gástricas, con la finalidad de mejorar las condiciones de salud de la población huancavelicana y, por ende, de disminuir la carga de morbilidad crónica y mortalidad entre la misma.

\section{REFERENCIAS BIBLIOGRÁFICAS}

1. Tapia GA. Incidencia: concepto, terminología y análisis dimensional. Programa de Publicaciones. Organización Panamericana de la Salud (OPS/OMS). Washington,D.C EE.UU. [Internet] 2019. [Consultado 2019 Sep 15] Disponible en: https://www.researchgate. net/publication/242150483_Incidencia_concepto_ terminologia_y_analisis_dimensional

2. Valdivia RM. Gastritis y Gastropatías. Revista Gastroenterología de Perú. [Internet]. 2011; 31(1): 38-48 [Consultado 2019 Nov 04] Disponible en: http://www. scielo.org.pe/pdf/rgp/v31n1/a08v31n1

3. Louis PC, Quintero I, Gutiérrez CP. Guía Latinoamericana de Dispepsia Funcional [Internet] 2014;44(2) [Consultado 2019 Abr 27] Disponible en: https://url2.cl/CeTtU

4. ProMEDmail. International Society for Infectious Diseases. (PRO/PORT> Novo coronavírus, COVID-19 Brasil (11) (SP, ex-Itália), primeiro caso provável. vol. 2020.

5. Prochazka ZR, Salazar MF., Barriga CE, Salazar CF. Prevalencia de Helicobacter pylori en una clínica privada de Lima: sensibilidad de las biopsias del antro y el cuerpo, y la prueba rápida de la ureasa. Rev. gastroenterol. Perú [Internet] 2010; 30(1): 33-39 [Consultado 2019 Jun 05] Disponible en: http://www.scielo.org.pe/scielo.php?script $=$ sci_arttext\&pid=S1022-51292010000100005\&lng=es

6. Barreda CC, Arcana LR, Salazar CF, Barriga CE, et al. Lower prevalence of Helicobacter pylori infection observed in patients with erosive esophagitis. Revista Gastroenterología de Peru. [Internet] 2014; 34(1):33-7 
[Consultado 2019 Jun 12] Disponible en: http://www. scielo.org.pe/pdf/rgp/v34n1/a05v34n1.pdf

7. McColl K. Helicobacter pylori infection. The New England journal of medicine [Internet] 2010; 362: (1); 597-604 [Consultado 2019 Oct 11] Disponible en: https://pubmed.ncbi.nlm.nih.gov/20427808

8. Domínguez RR, Huanca PA. Prevalencia de infección por $\mathrm{H}$. Pylori en una población de nivel socioeconómico medio y alto. Revista Médica. La Paz [Internet] 2013; 19(1): 35-39. [Consultado 2019 Nov 24] Disponible en: http://www.scielo.org.bo/scielo.php?script=sci_ arttext\&pid=S1726-89582013000100006\&lng=es

9. Pareja CA, Navarrete MP, Parodi GJ. Seroprevalencia de infección por Helicobacter pylori en población adulta de Lima, Perú 2017. Horizonte Medicina [Internet] 2017; 17(2): 55-58. [Consultado 2019 Abr 14] Disponible en: http://dx.doi.org/https://doi.org/10.24265/ horizmed.2017.v17n2.8

10. Ramírez RA, Chinga AE, Mendoza RD, Leey $C J$, Segovia CC, Otoya C. Variación de la prevalencia del H. pylori en el Perú período (1985-2002), en una población de nivel socioeconómico medio y alto. Revista gastroenterología de Perú [Internet] 2003; 23(2): 92-98 [Consultado 2019 Jul 23] Disponible en: http://www.scielo.org.pe/scielo. php? script=sci_arttext\&pid=S1022-512920030002000 $02 \& \operatorname{lng}=e s$
11. Ozaydin N, Turkyilmaz SA, Cali S. Prevalence and risk factors of Helicobacter pylori in Turkey: a nationally representative, cross-sectional, screening with 13C-urea breath test. BMC Public Health [Internet] 2013;13(1): 215 [Consultado 2019 Jul 12] Disponible en: https://www. ncbi.nlm.nih.gov/pmc/articles/PMC3880349/

12. Replogle M, Glaser S, Hiatt S, Parsonner J. Biologic sex as risk factor for Helicobacter pylori infection in healthy young adults. Am J Epidemiologia [Internet] 1995;142(8): 856-63 [Consultado 2019 May 03] Disponible en: https:// doi.org/10.1093/oxfordjournals.aje.a117725

13. Jara-Romero L, Sánchez-Figueroa C, Santana-Bazalar $D$, León-Jiménez F, Cubas-Benavides F. Frecuencia de Helicobacter pylori y características clínicas en niños con endoscopía digestiva alta de un hospital de Lambayeque: 2007-2010. Rev Cuerpo Med HNAAA. [Internet] 2013; 6(3): 28-32 [Consultado 2019 Feb 15] Disponible en: https://dialnet.unirioja.es/descarga/ articulo/4687220.pdf

14. Magalhães QD, Mayuko SG, Aguiar RA, Camargos RF, et al. Helicobacter pylori infection in infants and toddlers in South America: concordance between [13C]urea breath test and monoclonal $\mathrm{H}$. pylori stool antigen test. J Clin Microbiol [Internet] 2013; 51(11): 37-40 [Consultado 2019 Jun 22] Disponible en: https://jcm.asm.org/content $/ 51 / 11 / 3735$ 